\title{
Lexis
}

Journal in English Lexicology

$8 \mid 2014$

Metaphor Studies in the English Language

\section{Argots, métaphore et « effet de loupe »}

\section{Fabrice Antoine}

\section{OpenEdition}

\section{Journals}

Édition électronique

URL : http://journals.openedition.org/lexis/311

DOI : 10.4000/lexis.311

ISSN : 1951-6215

\section{Éditeur}

Université Jean Moulin - Lyon 3

\section{Référence électronique}

Fabrice Antoine, «Argots, métaphore et « effet de loupe » », Lexis [En ligne], 8 | 2014, mis en ligne le 09 janvier 2014, consulté le 19 avril 2019. URL : http://journals.openedition.org/lexis/311; DOI : 10.4000/ lexis.311

\section{(9) $(\mathbb{\Theta \Theta}$}

Lexis is licensed under a Creative Commons Attribution-NonCommercial-NoDerivatives 4.0

International License. 


\title{
Argots, métaphore et « effet de loupe »1
}

Fabrice Antoine ${ }^{2}$

\section{Résumé}

On tente ici de répondre à la question "comment naissent les métaphores ? ", à la lumière de la création lexicale dans les argots. Un certain nombre d'analyses (F. Rastier, U. Eco, M. Le Guern, G. Lakoff \& M. Johnson, etc.) intègrent la notion de parcours du sémème, préalable au saut métaphorique, qui semble peu compatible avec les caractéristiques nécessaires des argots, d'où la proposition d'explication des équations métonymique et métaphorique par un mécanisme que l'on baptise "effet de loupe ». L'autopsie sémantique d'une métaphore presque vive des argots français et l'analyse d'un corpus d'argot anglais permettent de montrer le fonctionnement de cet effet et de souligner le lien entre métonymie et métaphore, qui sont les deux matrices sémantiques essentielles de la lexicogenèse argotique.

Mots-clés : métaphore - métonymie - argots - analyse componentielle - lexicogenèse « effet de loupe»

\begin{abstract}
This paper attempts to answer the question "How are metaphors born?", as far as lexical creation in slang is concerned. Quite a number of theorists (e.g. F. Rastier, U. Eco, M. Le Guern, G. Lakoff \& M. Johnson) posit that sememes are thoroughly examined prior to the isolation of one seme for the creation of a metaphor, which appears not to be compatible with the characteristics of slang; this suggests another mechanism is at work here, one for which the name "magnifying-glass effect" is proposed. How this works is described in the "autopsy" of an almost live metaphor of French slang and in the analysis of a corpus of English slang words and expressions; these also stress the strong link between metonymy and metaphor, which are indeed the two essential semantic processes at work in slang.
\end{abstract}

Key-words: metonymy - metaphor - slang - word-formation processes - "magnifyingglass effect"

\footnotetext{
${ }^{1}$ Je souhaite remercier ici Ronald Jenn (Université Lille 3) et Pierre Labrosse (Université Paris 4) pour leur lecture attentive et leurs suggestions ; également Cosimo De Giovanni (Université de Cagliari) qui a été à l'origine d'une étude antérieure (Antoine [2011]), que celle-ci prolonge et tente d'approfondir.

${ }^{2}$ Université Lille 3 - Charles de Gaulle, France : fabrice.antoine@univ-lille3.fr
} 


\section{Introduction}

On admet généralement aujourd'hui, et ce, depuis un bon nombre d'années, que la métaphore n'est pas qu'un trope parmi d'autres, à disposition par exemple du poète, du prosateur ou de l'orateur pour lui permettre de travailler et enjoliver son discours - elle est structurante de l'appréhension, de la vision et de la description du monde par l'homme : elle est structurante du lexique et, partant, du langage.

[La métaphore] est un des mécanismes fondamentaux par lesquels l'esprit humain est capable d'appréhender l'univers. Le système conceptuel humain est de ce fait entièrement structuré et défini par un réseau de métaphores. [Lehmann 1998 : 90]

Telle est la conclusion d'un chapitre sur la métaphore abondamment nourri de la recherche sur cette figure de la deuxième moitié du XXe siècle. Dans La Métaphore, Jacques Dürrenmatt souligne que

[...] beaucoup sont d'accord pour considérer désormais que le phénomène métaphorique [...] n'est pas marginal mais peut au contraire constituer le pivot autour duquel s'organiseraient ou pourraient s'organiser aussi bien le langage en lui-même qu'un certain nombre de discours spécialisés » [Dürrenmatt 2002 : 7].

On comprend donc le regain d'intérêt pour la métaphore, mais l'on rappellera que, dès le début du XXe siècle, par exemple avec Gaston Esnault, l'on percevait cette fonction essentielle de la métaphore dans la « sémantique populaire » (Esnault [1925]).

On tente, depuis Aristote au moins (et peut-être faut-il penser avec Umberto Eco que rien de nouveau n'a été dit depuis ?3), de décrire le mécanisme de la métaphore, de le démonter pour l'expliquer, et sont proposés des raisonnements souvent bâtis à partir de métaphores isolées, du type "loup = homme», etc. On tente de décrire le fonctionnement de cette figure par celui de quelques métaphores... mortes, par la force des choses, ce que Botet [2008: 57] appelle « une autopsie sémantique ». Il faudrait sans doute des 'Think Aloud Protocols' (TAP) ou «protocoles de verbalisation » pour déceler ce qu'il advient lorsque se crée une métaphore dans l'esprit d'un écrivain par exemple, mais il faudrait pouvoir les adapter pour être en mesure d'observer ce qu'il en est lorsque des métaphores se créent spontanément, à l'oral par exemple, dans le cerveau, dans la bouche, du locuteur ordinaire. En l'absence de tels moyens d'observation, nous en sommes réduits à examiner les traces, les indices, les signes qui subsistent dans des métaphores lexicalisées ou, si nous avons de la chance, presque lexicalisées. Ne peut-on pas, en raisonnant sur les étoiles mortes, émettre des hypothèses sur la façon dont elles sont nées? En d'autres termes, la question posée est bien : comment naissent les métaphores?

\footnotetext{
3 « of the thousands and thousands of pages written about metaphor, few add anything of substance to the first two or three fundamental concepts stated by Aristotle» (Eco [1983: 217-18]).
} 


\section{Le saut métaphorique, avec ou sans parcours?}

L'analyse componentielle fournit le cadre dans lequel se situe la présente étude; c'est sans doute l'approche la plus convaincante du point de vue sémantique, comme le souligne Botet [2008 : 29 sqs], pour qui elle se retrouve sous diverses formes dans les théories modernes de la métaphore; il souligne par exemple les similitudes entre François Rastier et Umberto Eco (Botet [2008 : 48]). Avant Rastier, Le Guern [1973 : 15] avait posé que le mécanisme de la métaphore s'explique par «la suppression, ou plus exactement par la mise entre parenthèses d'une partie des sèmes constitutifs du lexème employé »; pour lui, la métaphore du substantif "fait intervenir une suspension sémique portant sur les éléments que Greimas appelle les sèmes nucléaires » (Le Guern 1973: 19]). Après lui, Martin [1983: 219], qui rappelle que pour Paul Ricoeur, la métaphore " consiste à 'oublier', à éliminer - proprement à faire abstraction de... », va dans le même sens, et tient que la création métaphorique consiste à sélectionner dans les prédications propres à un objet celle qui convient également à un prédicat donné et "éliminer transitoirement les autres ». Ce qui précèderait donc l'apparition d'une métaphore, c'est le parcours du sémème de deux objets (les deux termes de l'équation métaphorique) suivi de l'effacement, la "mise entre parenthèses ", d'une partie de leurs sèmes. La sélection d'un ou de plusieurs sèmes (idéalement un seul, selon Umberto Eco, comme le rappelle Botet [2008: 30], ne peut s'opérer qu'au terme d'un parcours du sémème. On ne dirait donc que l'homme est un loup, pour reprendre l'exemple de Martin, qu'au terme du parcours du sémème de «loup » (sous forme de prédications : le loup est un mammifère, ... est un canidé, ... est carnivore, ... ressemble à un grand chien, ... hurle, ... est sauvage, ... vit dans les forêts, ... vit en meute, etc.) et de celui de «homme»; or, on se rend bien compte que, dans cette liste, manque un sème qui n'apparaîtra pas dans une définition " objective » de dictionnaire mais qui sous-tend, justifie, la métaphore : le trait |sanguinaire| ou |cruel| - qui est celui-là même qui fonde le mythe du loup des Fables de La Fontaine ou des contes, de Perrault ou d'autres, en tout cas pour la culture française.

Ce type d'observation, que l'on peut répéter à l'envi, à partir de métaphores de la langue usuelle, conjugué à l'étude des argots, qui sont éminemment langues de déplacement ${ }^{4}$ et font un usage intensif de la métaphore (et de la métonymie), amène à remettre en question cette notion de parcours de sémèmes dans le mécanisme métaphorique. Étant donné le caractère oral des argots, et - la plupart du temps spontané, immédiat, non réfléchi, non raisonné, le parcours puis effacement de sèmes paraît trop coûteux (en opérations mentales et en temps) pour être adapté à la création métaphorique foisonnante et souvent instantanée parmi les argotophones. J'ai donc tenté de décrire un processus différent, moins onéreux, plus rapide, qui consiste en la mise en relief ou au premier plan d'au moins un sème, qui est le seul que voit ou veut voir le locuteur d'un objet donné, qui s'impose à lui en quelque sorte ou qu'il privilégie dans sa vision de cet objet. Pour ce locuteur, tel objet n'est que ce sème-là - ce qui, évidemment, donne d'abord un caractère très idiosyncrasique à la métaphore, qui se

\footnotetext{
${ }^{4}$ Ce sont strictement des lexiques et non des langues, bien sûr. Cette étiquette est utilisé par commodité, comme on parle d'argot (au singulier) plutôt que des argots, en entendant bien la pluralité de ces lexiques. Voir Antoine [2008].
} 
lexicalisera d'autant plus aisément, rapidement, que d'autres pourront partager cette vision « réductrice » : si pour plusieurs locuteurs, le sème |sanguinaire| est celui auquel se réduit le «loup » et aussi l' «homme», alors la métaphore "l'homme est un loup » sera validée et se lexicalisera. $\mathrm{Si}$, de la même façon, le sème |sert de soutien| résume aussi bien "ami » que "poteau » (la pièce de charpente : les argots puisent à pleines mains dans les langues de métier), alors « poteau » pourra désigner un ami ${ }^{5}$.

Le transfert ou saut métaphorique est possible lorsqu'il y a coïncidence de deux objets avec le même sème haussé ou privilégié. Le même sème peut être ensuite privilégié dans d'autres objets, par une sorte de mimétisme, ce qui amène d'autres métaphores en cascade - il n'est pas toujours aisé de retrouver la métaphore " origine », mais cela fait le mystère du lexique, en particulier argotique. Par exemple, si, en argot, le sème de «pistolet » qui s'impose comme descripteur unique, suffisant, de l'objet est |émet un bruit détonant|, "pétard", qui possède ce sème, peut donc désigner un pistolet. Si le sème privilégié est, pour un autre locuteur, lémet un bruit semblable à un aboiement|, donc, pour résumer, |aboie|, (et l'attrait d'un jeu de mots sur «chien » n'est évidemment pas exclu), alors « aboyeur » (chien qui aboie, avant d'être métaphorisé en « homme qui crie/aboie »), possédant le même sème, peut désigner un pistolet - et, par suite, "Azor", qui est une sorte, un archétype, d'aboyeur, donc possède lui aussi le même sème, le peut aussi... et donc, tout objet qui possède ce sème en a aussi la

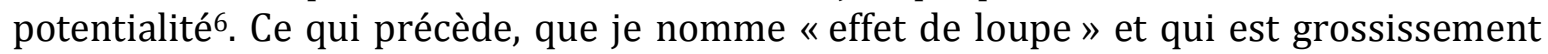
d'un trait au détriment des autres ( $c f$. aussi Antoine [2011]), obéit au principe du moindre effort à l'œuvre dans la langue, en particulier dans les argots et dans la langue familière, qui fait que, par exemple, le moindre coût articulatoire est préféré (troncation, syncope, effacement d'éléments syntaxiques, etc.) et qui va dans le sens de la spontanéité de la création lexicale ${ }^{7}$. Celle-ci va à l'évidence de pair avec l'immédiateté de l'expérience du monde, sur laquelle se fonde la métaphore, quel qu'en soit le domaine ; Lakoff et Johnson [1985: 30] l'ont clairement posé: «Nous avons le sentiment qu'aucune métaphore ne peut jamais être comprise ou même adéquatement représentée indépendamment de son fondement expérientiel » - mais Esnault [1925: 12] parlait, pour comprendre et expliquer les métaphores, de l'«inspiration prise des choses à exprimer ; [...] la probable réaction de telle sorte de sujets selon les dates, les métiers », ce qui, on en conviendra, fait bien référence à cette même expérience du monde.

\footnotetext{
5 Dès 1259 selon le Trésor de la langue française (TLF), 1400 («posteau ») selon Esnault [1965]. Donne « pote » par apocope au XIXe siècle (1898-99).

${ }^{6}$ Cela est non négligeable dans une perspective de prédiction des créations lexicales en argot et dans une autre, de traduction des argots.

${ }^{7}$ Le « principe du moindre effort » fait en général référence à la loi de Zipf, du nom du linguiste américain qui s'est intéressé en particulier à la fréquence des mots en langue dès 1935. Cette loi a été adaptée notamment en phonologie. On souligne que Gaston Esnault, dans le cadre de sa réflexion sur les métaphores et leur éclosion, la pose comme allant de soi dans l'exposition de la vraisemblance de ce qu'il nomme « sématisme » (c'est-à-dire «ce qu'il y a dans la pensée du sujet au moment où il crée un mot ») : «La vraisemblance [...], c'est l'économie explicative faisant toute dépense de matériau nécessaire, mais alléguant le principe du moindre effort jusque dans les tâtonnements » (Esnault [1925 : 12]). L'apparition de cette expression ici dix ans avant Zipf (et 24 ans avant la publication de son ouvrage Human Behaviour and the Principle of Least Effort, Oxford, Addison Wesley Press, 1949) est pour le moins intéressante.
} 
Soulignons par ailleurs que l'effet de loupe a d'abord été défini comme alternative à l'explication du mécanisme métaphorique décrite plus haut. J'ai constaté ensuite qu'Albert Henry, dans Métonymie et métaphore, fait de ces deux figures une analyse qui semble hésiter entre parcours + effacement et ce qu'il nomme focalisation, en particulier pour ce qui concerne la métonymie, qu'il décrit de la façon suivante: «l'usager a considéré d'une façon toute particulière certains des caractères inclus dans la définition du terme, [et] il a fini par ne plus retenir que ceux-là » (Henry [1971 : 20]). Il ajoute :

Qu'il s'agisse de métonymie ou de synecdoque, l'esprit joue sur la contiguïté entre certains concepts, sur les rapports entre concepts, en faisant abstraction, ou en feignant d'ignorer certains éléments de la compréhension véritable. Voile pour vaisseau : l'esprit éclaire vivement cette caractéristique du navire, voile les autres et utilise ensuite le terme désignant la partie, pour évoquer le tout. [Henry 1971 : 23].

Henry ne parle qu'ensuite, et rapidement, de champs sémiques, dont il souligne qu'ils restent extensibles car l'esprit peut considérer comme important ce qui est secondaire, indiquant par là que la métonymie, mais aussi la métaphore, peut s'appuyer sur un sème afférent (Martin) ou connotatif (Pottier [1964]), tout comme dans l'effet de loupe. Il avance ensuite une nouvelle définition de la métonymie où apparaissent les notions clés chez lui :

En métonymie, l'esprit, parcourant un champ sémique, focalise sur un des sèmes et désigne le concept-entité qui est l'objet de sa contemplation par le mot qui, en pure réalité linguistique, exprimerait ce sème, quand il est considéré en tant que conceptentité. » [Henry $1971: 25$; mes italiques].

Il établit alors un lien très fort entre métonymie et métaphore qui rejoint ce que je nomme ici « effet de loupe » : "La métaphore superpose deux concepts dont une portion seule est mise tout à fait au point par la double focalisation métonymique : une portion seule, c'est-à-dire en général un sème. » [Henry 1971 : 67] La notion de parcours, double ou simple, a disparu ici et la métaphore de la focalisation privilégie une notion de vision : vision, visualisation, d'un objet d'une manière particulière, qui n'accorde d'importance qu'à un élément, le seul retenu pour représenter l'objet. Henry ne montre pas en quoi le mécanisme qu'il décrit est plus économique, plus spontané qu'un autre, ne l'oppose pas au parcours + effacement; c'est ce que le concept d'effet de loupe tente de faire. L'analyse de deux exemples qui suit vise à le montrer à l'œuvre dans le fonctionnement de ce que Calvet [1994] appelle « machines à créer du lexique », qui sont pour l'essentiel des machines à métaphores et métonymies.

\section{De l'autopsie sémantique d'une métaphore presque vive}

Le premier exemple sera d'un mot isolé, «hibiscus", que j'ai déjà brièvement abordé ailleurs (Antoine [2011]), et qui me semble intéressant en tant que c'est, argotiquement, une métaphore pas tout à fait morte : Pierre Perret fait figurer ce mot dans son Nouveau petit Perret illustré par l'exemple de 1982, dans la locution «se 
caresser l'hibiscus ou se chatouiller l'hibiscus » (classée à la lettre C) avec le sens : "pour une femme, se masturber ». Cette expression n'apparaît pas, bien sûr, dans le Dictionnaire érotique moderne d'Alfred Delvau de 1864 (où l'on vérifie par acquit de conscience), non plus, et c'est plus significatif, que dans le Dictionnaire érotique (dont le titre complet est Dictionnaire historique, stylistique, rhétorique, étymologique de la littérature érotique) de Pierre Guiraud, de 1978 - celui-ci enregistrant, par exemple, "jouer de la mandoline», dans le même sens, que Pierre Perret donne aussi. Ce qui précède tend à montrer que Perret mêle les expressions et sens attestés de l'argot, plus ou moins moderne, à des expressions et sens qui lui sont personnels, qui sont des créations métaphoriques au gré de ses textes et chansons. Le Dictionnaire des gros mots de Patricia Vigerie (2004) relève que «les expressions illustrant la masturbation féminine sont très peu nombreuses comparées à celles de l'homme " (Vigerie [2004: 41]) - ce qui ne devrait pas être étonnant si l'on a bien à l'esprit que les argots ont d'abord été lexiques d'hommes. Il est intéressant de citer cet ouvrage avant de revenir un peu en arrière dans l'histoire lexicographique de cet «hibiscus »: «En 1982 apparaissaient les locutions poétiques (se) chatouiller ou se caresser l'hibiscus, basées sur l'image d'une plante à grandes fleurs en cornet, probablement d'après la forme du corps de la femme » (Vigerie [2004 : 42]). Le dictionnaire de P. Perret apparaît dans la bibliographie, de même que le Dictionnaire de l'argot de Jean-Paul Colin et Jean-Pierre Mével, de 1990. C'est à cet ouvrage que l'on doit un figement (au sens de rigor mortis, pour poursuivre la métaphore de l'autopsie) de l'expression de P. Perret: J.-P. Colin et J.-P. Mével l'enregistrent, et la donnent comme validée, alors que la seule occurrence relevée est sous la plume de P. Perret en 1982 ! Voici leur article, sur lequel P. Vigerie s'est appuyée et qui confère à une métaphore vive, une sorte d'hapax, un statut de métaphore lexicalisée :

hibiscus $\mathrm{nm}$ se caresser ou se chatouiller l'hibiscus, se masturber, en parlant d'une femme.

ETYM. Locution métaphorique (plante à grandes fleurs en cornet), à prétention poétique - 1982 [Perret]

Ajoutons que Le Dico du sexe d'Albert Doillon [2002], s'il donne "se jouer un air de mandoline » et «jouer de la mandoline » (ex. Laccassagne [1928]), ne mentionne cet " hibiscus » nulle part, non plus que François Caradec, autant dans N'ayons pas peur des mots [1988] que dans Dictionnaire du français argotique et populaire [2009].

L'intérêt de cette métaphore réside dans son caractère individuel et " émergent ", c'est-à-dire que nous la saisissons quasiment au moment où elle se forme dans l'esprit de son créateur, auteur de chansons et familier de la création métaphorique, «à prétention poétique » ou non. P. Perret a-t-il parcouru le sémème de « hibiscus » pour en « mettre entre parenthèses » ou " éliminer transitoirement » une partie des sèmes ? A-til passé en revue les traits caractéristiques de l'objet « hibiscus », les éliminant un à un jusqu'à sélectionner celui ou ceux qui convien(en)t, ou le processus peut-il être envisagé de manière plus spontanée et économique? On notera l'embarras de J.-P. Colin et J.-P. Mével et celui de P. Vigerie pour expliciter la métaphore : les premiers, en réalité, n'expliquent rien et font référence à des "grandes fleurs en cornet» (qui peuvent 
évoquer le lys ou le liseron mais sans doute pas l'hibiscus !) ; la seconde, embrayant sur l'image du « cornet », la relie on ne sait comment à la « forme du corps de la femme».

A la décharge de ces auteurs, l'hibiscus est un arbre tropical (ce serait proprement un petit arbre ou un arbuste d'ailleurs), qui n'appartient pas d'emblée au domaine expérientiel du locuteur européen. Les lexicographes français peinent à produire une définition : " arbre tropical à fleurs ornementales, dont une espèce est l'ambrette et dont une autre fournit un textile. (Famille des malvacées). " dit le Petit Larousse 1994; "arbre tropical à grandes fleurs de couleurs vives", dit le DIXEL 2010. Les lexicographes britanniques (Collins) ou américains (Webster) ont les mêmes difficultés. Le sémème de "hibiscus", métonymique pour "fleur d'hibiscus", apparaît ici bien pauvre : on y retient quatre sèmes, |fleur|, |tropicale|, |grande|, |de couleurs vives|, qui ne sont pas d'un grand secours pour visualiser la fleur en question. Or, l'équation métaphorique est "sexe de la femme = (fleur d')hibiscus", pour que "se masturber, pour une femme = se chatouiller l'hibiscus ». Mon hypothèse est que, pour que cette équation se mette en place spontanément et immédiatement, deux images mentales se superposent, celle du sexe de la femme et celle de la fleur d'hibiscus, toutes deux issues de l'expérience du locuteur (expérience qui peut aussi être fantasmée, mais peu importe) et que l'un des traits de l'un et de l'autre passe au premier plan, s'impose comme le seul pertinent, sans même que les autres traits ne soient envisagés : ce trait est haussé, mis en relief, dans l' " effet de loupe »; il est l'objet, au détriment de tous les autres traits. Ici, l'expérience (c'est-à-dire la connaissance de la fleur d'hibiscus, qui permet de la visualiser) amènerait à un sémème de ce type : |fleur|, |tropicale|, |grande|, |tons rosés|, |pistil allongé|, |pistil dressé|, dans lequel les deux derniers traits, pour cette métaphore, sont vus comme les éléments essentiels, uniques, superposés à deux sèmes du sémème de " clitoris » : |petit organe|, |de la femme|, |érectile|, |allongé|, |très sensible $\left.\right|^{8}$. Les sèmes haussés sont-ils centraux ou inhérents (c'est-à-dire faisant partie des traits définitoires essentiels) ou périphériques ou afférents (c'est-à-dire non essentiels à une définition lexicographique minimale ${ }^{9}$ ) ? Ici, ils semblent être périphériques, ce qui souligne que le locuteur, lors du processus de création métaphorique, peut privilégier n'importe quel trait, même très marginal, voire un sème périphérique pour l'un des termes de l'équation et un sème central pour l'autre - ce qui explique aussi pourquoi tant de métaphores mortes sont difficiles, voire impossibles, à expliciter.

J'ajouterais que ce saut métaphorique, du sexe de la femme à la fleur d'hibiscus, possible uniquement si les deux termes de l'équation font partie du domaine expérientiel du locuteur, aurait très bien pu se faire vers une autre fleur ou un autre objet que le locuteur, P. Perret, aurait convoqué de sa mémoire. Les mêmes sèmes auraient pu actualiser d'autres images, par exemple, pour rester dans le domaine (après tout un tant soit peu poétique) de la flore, ils auraient pu entraîner la visualisation d'un arum ou d'un anthurium, fleurs à pistil exhaussé. Cela souligne que d'autres facteurs interviennent certainement dans l'équation, qui sont, peut-être, visualisables, comme la forme de la fleur, sa couleur (le blanc de l'arum, le rouge profond de l'anthurium, etc.) ou

\footnotetext{
8 Sémème établi en croisant les définitions de plusieurs dictionnaires : Petit Larousse, DIXEL, Collins English Dictionary.

${ }^{9}$ Celle-ci, dans le DIXEL ou le Petit Larousse est-elle suffisante d'ailleurs?
} 
sont plus abstraits, comme la longueur et la consonance du nom de l'objet: est-il significatif que « hibiscus » et «clitoris » aient tous les deux trois syllabes, se terminent par [s] et comportent la syllabe [is] ? La présence du [i] deux fois dans les deux mots influe-t-elle sur le choix? La finale en «-um » des deux autres noms mentionnés ici les disqualifie-t-elle par ses consonances? Autant de questions qui peuvent se poser et d'éléments dont la superposition ou la confluence favorise sans aucun doute la naissance, l'éclosion, d'une métaphore.

Un court exemple complémentaire me servira de transition vers l'ensemble cohérent de métaphores que je souhaite décrire et exploiter. Il s'agit du mot « cocotteminute ${ }^{10}$, qui, dans le jargon des policiers, désignait ce que la terminologie administrative appelait "piédestal de circulation", qui est une |plate-forme|, |circulaire|, |surélevée|, |sur laquelle se tient un agent de police|, |pour régler la circulation automobile|: les sèmes centraux sont ici mis en évidence, des sèmes périphériques étant |installée au milieu d'un carrefour| et |rouge et blanche|. Rien qui explique à première vue le saut métaphorique entre les deux objets, sauf que les traits associés dans la visualisation de ce piédestal de circulation ont vraisemblablement été, dans l'imaginaire gouailleur du créateur de cette métaphore, |siffle| et |tourne| parce que c'est ce qui décrit le plus succinctement et le plus spontanément l'activité de l'agent affecté là, à quoi il faut ajouter la chaleur (un des facteurs qui justifiait de relever plusieurs fois par heure les agents à la circulation à la place de la Concorde à Paris) et la forme cylindrique de l'objet, qui évoque un récipient. On voit que les sèmes convoqués appartiennent très lointainement au piédestal, mais sont spontanément associables à l'ustensile de cuisine. En quelque sorte, en voyant le policier tourner et siffler, la réponse spontanée à la question « qu'est-ce qui tourne et siffle ? » permet le saut métaphorique vers la cocotte-minute, sans parcours et sélection de sème(s) mais en "grossissant » un/des sème(s) de l'un ou l'autre objet : effet de loupe, une nouvelle fois. ${ }^{11}$

\section{Effets de loupe et matrices lexicogénétiques}

Je voudrais montrer maintenant l'effet de loupe à l'œuvre dans un corpus ${ }^{12}$ des désignations du policier en argot et langue familière en anglais, du XVIe siècle à nos jours (anglais britannique et américain). Ainsi que je l'ai observé ailleurs à partir d'un corpus similaire en français, toutes les désignations du policier se rangent dans un nombre fini de matrices formelles, mais aussi sémantiques. Ces dernières résultent d'un effet de loupe, d'une mise en relief d'un trait du policier qui en vient à n'être que ce trait. On comprend fort bien que, dans la spontanéité, l'immédiateté, de la création lexicale en argot, le créateur ne parcourt pas une définition du policier pour en sélectionner un trait

\footnotetext{
10 On n'abordera pas ici le sens argotique répertorié de "prostituée qui fait de l'abattage ».

11 Est-il indifférent par ailleurs que «cocotte» et "poulet» se rejoignent dans un autre champ sémantique ? Sans doute pas, en renfort de l'effet de loupe.

12 On me permettra d'utiliser ici, par commodité au moins, le mot 'corpus' dans le sens d'« ensemble de mots utilisé à fins d'analyse », étant entendu que ces mots sont ici dissociés de leur contexte, le corpus de dictionnaires et de textes argotiques dont ils sont extraits, et donc que 'corpus' est pris dans le sens de ce que Rastier [2002] appelle « sac de mots ».
} 
à partir duquel élaborer une métaphore : le caractère répétitif de la mise en relief d'un nombre limité de sèmes me semble indiquer que la création métaphorique s'appuie sur un ensemble d'équations de base (du type : « policier = celui qui porte un uniforme » ou «policier = délateur » ou encore " policier = archétype de policier ») qui sont sollicitées et permettent des sauts métaphoriques vers des objets vus comme typiquement porteurs du même sème, comme n'étant plus que ce trait, se résumant à lui.

Le corpus lexical dont il s'agit comporte 316 items qui désignent de façon argotique ou familière un policier, aussi bien un policier en uniforme (constable) qu'un inspecteur de police ou la police en général, en anglais britannique ou américain, à toutes époques (le mot le plus ancien date de 1593 au moins et le plus récent de la fin du XXe siècle. Il a été collecté en croisant divers dictionnaires, comme, par exemple, A Concise Dictionary of Slang and Unconventional English (From A Dictionary of Slang and Unconventional English by Eric Partridge de Paul Beale, The Concise New Partridge Dictionary and Unconventional English de Tom Dalzell et Terry Victor, le Dictionary of American Slang de Harold Wentworth et Stuart B. Flexner, The Oxford Dictionary of Slang de John Ayto, mais aussi des dictionnaires d'argot anciens que l'on trouve en fac-similé sur Internet ou des lexiques dont on a établi un bon degré de fiabilité par divers filtres. Chaque item a été validé par deux sources au moins.

Rappelons brièvement comment les outils utilisés pour ordonner et représenter le corpus ont été élaborés. En s'appuyant sur les matrices lexicogénétiques formelles définies par Tournier [1985] pour rendre compte du lexique anglais, auxquelles on adjoint deux matrices que l'on a définies spécifiquement pour rendre compte de particularités des argots (cf. Antoine [2004] \& Antoine [2008]), on obtient un ensemble de douze modes de formation lexicale qui sont: préfixation, suffixation, dérivation inverse, composition, amalgame, onomatopée, conversion, troncation, siglaison, emprunt, déformation et jeu de mots (sur proximités phoniques ou graphiques). Ces matrices constituent l'axe formel de la «machine à créer du lexique » par quoi je tente d'objectiver le processus intellectuel ou mental par lequel les mots d'argot sont générés. L'autre axe est celui des matrices sémantiques, qui sont des équations entre deux objets, de type métonymique ou métaphorique. Si les matrices formelles forment un ensemble fixe et clos, les matrices sémantiques sont à définir pour chaque ensemble lexical, comme ici les désignations du policier, à partir de l'analyse des sémèmes de chaque mot. Elles sont donc les procédés de remotivation des mots, sans lesquels les argots seraient bien pauvres. Elles permettent l'écriture d'une définition la plus exhaustive qui soit d'un objet, en faisant apparaître un ensemble de sèmes, centraux et périphériques, que la définition lexicographique standard ne présente pas. Les matrices des deux catégories en combinaison ou non permettent de rendre compte de la totalité des items tels que ceux réunis pour cette étude; cela fait apparaître clairement que le lexique argotique n'est pas un ensemble anarchique et foisonnant, mais que sa formation présente des régularités qui éclairent en retour le fonctionnement du procédé métaphorique.

Ici, pour le policier, la définition que l'on pourrait bâtir, à partir des traits saillants de l'« objet » haussés par les argotophones, pourrait être la suivante, organisée en deux parties, la forme de l'objet, au sens le plus large, et sa fonction : 
Le policier se distingue par l'éventuel port d'un uniforme, qui peut avoir une couleur dominante précise, et celui d'un couvre-chef, l'utilisation de certains accessoires liés à sa fonction; son mode de déplacement est la marche ou alors il utilise certains véhicules éventuellement reconnaissables à des éléments précis; il est réputé être d'une intelligence moyenne, qui confine à la bêtise, et il est sensible à la corruption.

Il a trois fonctions principales: celle de surveiller (veiller mais aussi épier: ce qui mobilise son œil ou son nez/odorat et le rend assimilable à un délateur ou un traître), celle de rechercher/arrêter (ce qui peut se traduire aussi par frapper) et enfin celle de protéger.

La définition lexicographique usuelle (Petit Robert pour le français, Collins English Dictionary pour l'anglais) ne présente pas ces sèmes, plutôt périphériques, mais met en avant les sèmes |maintient l'ordre|, |assure la sécurité|, |recherche des criminels|, |exécute des arrêts judiciaires|, |réprime des infractions|, dont certains se retrouvent, de façon réduite et connotée, dans les fonctions du policier pour l'argotophone. Une matrice complémentaire, à mi-chemin entre forme et fonction par le sémantisme des mots qu'elle regroupe, est celle qui se traduit par l'équation "policier = archétype de policier » où un nom propre ou assimilé est choisi comme désignation du policier car il est porteur d'un ou plusieurs sèmes de forme ou de fonction, qui apparaît (-aissent), est/sont haussé(s), comme sa quintessence.

On a parlé d'équations métonymique et métaphorique, et de forme et de fonction de l'objet, ici, le policier : c'est que le premier type d'équation exprime la forme, tandis que le second dit la fonction. La première se fonde sur des éléments tangibles, immédiatement perceptibles, de l'objet, ou ressentis comme tels, non seulement physiques mais aussi moraux; pour le corpus lexical étudié ici, on peut les classer comme dans la figure 1 ci-dessous. Naturellement, n'apparaissent dans cette figure que les sèmes qui ressortent de l'examen des items qui sont la réalisation de l'équation métonymique, qui sont ceux "grossis », "haussés », par l'effet de loupe, qui sont donc les seuls pertinents aux yeux des créateurs de ces métonymies. ${ }^{13}$ Par exemple, contrairement au français, dans la sous-catégorie «uniforme» n'apparaît pas " chaussures », ou dans " accessoires » n'apparaît pas « insigne », parce que ces traits-là ne sont pas grossis comme ils le sont en français, donc ne sont pas perçus comme pertinents, comme définitoires du policier. Dans l'autre sens, ce sont les «traits de caractère » (bêtise, corruption - la péjoration est forte) qui ne sont pas "activés » en français. ${ }^{14}$

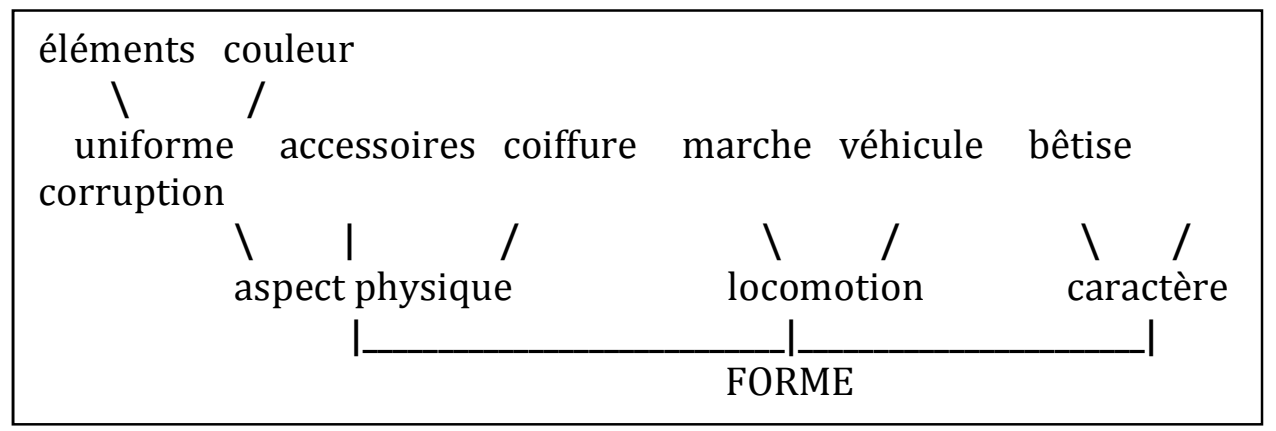

$13 c f$. Eco [1983: 251] : « only those semes or properties that the co-text has suggested as relevant ».

${ }^{14}$ Voir Antoine [2011] pour une analyse du corpus en français. 
La fonction se subdivise en trois catégories (communes à l'anglais et au français) : " surveiller», « recherche/arrêter » et « protéger »- qui sont les fonctions principales du policier vu au prisme de l'imagination collective dans le contexte anglo-américain. La matrice intermédiaire, "archétype », est, comme la "fonction », une équation du type métaphorique dans la mesure où il ne s'agit pas, comme c'est le cas de la forme, d'un simple effet de loupe (un sème grossi, haussé, se substitue au sémème entier ${ }^{15}$ ), mais plutôt d'un double effet de loupe, ou de la conjugaison de deux effets de loupe sur deux sémèmes différents.

Cela mérite que l'on s'y arrête : on pourrait arguer que le passage de policeman à pig se fait par un effet de loupe qui hausse le sème |trouve des choses cachées| d'abord dans le sémème de policeman puis par une sélection rapide d'objets dont le sémème contient le même sème, alors haussé à son tour pour la réalisation de l'équation métaphorique "policeman = pig ». Un autre cas serait le passage de policeman à copper $:$ un effet de loupe sur le sème |catches criminals| active la sélection d'objets présentant le même sème, puis vraisemblablement partie du sème, ou sème "plus large ", c'est-à-dire |catches|, qui, à son tour, déclenche l'activation des synonymes de catch : cop, nab, nail, snatch, grab, etc. Lors de la première activation, la métaphore peut aboutir avec nail-'em-and-jail-'em, qui fera partie du sémème du policier, en tant que devise du partisan du « tout répressif »; la deuxième activation débouche sur une impasse : peu de mots apparaissent, ou peut-être trop sont apparus, trop vagues, dont le sémème contient |catches|, sauf bogey ou beaksman (littéralement, " homme du juge », cf. " chien du commissaire » en français). Alors derrière les verbes synonymes activés apparaissent les noms qui désignent l'agent, implicites dans le verbe (un procès doit être conduit par un agent), lexicalisés pour certains (ex. nailer, snatcher), formés par suffixation en -ER ; copper, nabber, nailer, nickers, etc. contiennent tous dans leur sémème |catches| puisque leur définition est 'one that catches' - on a ici aussi deux effets de loupe successifs, avec pour ainsi dire une boucle de création lexicale où le lexème désignant l'agent est convoqué ou créé, actualisé.

Dans le cas de la matrice "archétype ", enfin, si l'on admet le modèle de "semiosis illimitée » (Eco [1983 : 238]) et son type d'analyse en forme d'encyclopédie (Eco [1983 : 229]) où tous les sèmes de l'objet seraient énumérés, ou du moins consignés, on peut admettre que le sémème le plus complet de policeman contiendrait un sème du type |personnage archétypal| ou |personnage stéréotypé| sous lequel seraient enregistrés tous les noms propres et assimilés de policiers devenus des archétypes dans la culture considérée; le français en a quelques-uns, propres (Cruchot, Schmitt, McCoy, Pandore, Starsky) ou non (Kojak) ; l'anglais en a une quantité, réels, fictifs, créés (Kojak, CHIPS, Dick, Lily Law, John Hop, statie, Keystone, Mulligan, Paddy, Uncle Bill, etc.). Le sémème de ces noms propres ou assimilés, autoréférentiels, est réduit à un élément différenciant qui est le nom lui-même : l'effet loupe hausse donc ce sème-là, commun avec la liste infinie de policeman.

\footnotetext{
${ }^{15}$ Eco [1983 : 244] écrit : « ... metonymy becomes the substitution of a sememe with one of its semes [...] or of a seme with the sememe to which it belongs ». Les exemples qu'il donne n'illustrent pas vraiment clairement la « substitution d'un sémème à l'un des sèmes » - celle d'un sème au sémème semble la plus cohérente. Par ailleurs, il ne se penche pas sur le mécanisme qui amène à cette substitution : sans parler de parcours et effacement, il semble cependant postuler un parcours du sémème avant la substitution.
} 
On visualise la machine à créer du lexique qui se définit ici en plaçant les matrices sémantiques sur un axe horizontal et les matrices formelles sur un axe vertical : les mots du corpus se rangent dans la grille ainsi formée en fonction des combinaisons entre formel et sémantique qui y sont apparentes (voir tableaux 1 et 2 en annexe). Tous les mots du corpus (316) entrent dans cette machine définie et finie, sauf 16 (soit 5\% du corpus), qui, pour la plupart, souffrent d'un déficit d'informations étymologiques qui empêche de les rattacher à une matrice sémantique ou formelle donnée.

Trente mots (soit 9,5\% du total) n'ont fait l'objet que d'un déplacement formel; ce sont surtout des résultats de conversion (de nom propre en nom commun, comme Ervine, Oliver ou Sam and Dave; d'adjectif en nom, comme odd ou bulky), d'emprunt (gendarme, garlo, musgro) ou de déformation (argots à clef divers, comme le rhyming slang (ducks and geese), le backslang (esclop, slop), le pig Latin (uzzfay) ou métathèse humoristique, faux fourchage de langue (occifer). On n'exclura pas pour certains, cependant, l'activation d'un trait sémantique en renforcement (par exemple pour ducks and geese: le rhyming slang est rarement non motivé (cf. Antoine [1999]) ; ou pour occifer, qui rime avec Lucifer, et esclop qui est télescopé en slop, qui se trouve avoir un sens connoté (comparer «dékon» pour "condé» ou «feuk» pour «feukeu», reverlanisation de "keuf», en français).

La conversion (en particulier de nom propre en nom commun et d'adjectif en nom) est d'ailleurs la matrice formelle la plus sollicitée, avec 51 items $(16,10 \%$ du corpus) : naturellement, le nombre élevé d'archétypes recensés en anglais, comme évoqué plus haut, explique cette prédominance. Le jeu de mots vient ensuite, qui se combine dans la quasi-totalité des cas avec une matrice sémantique et permet ainsi diverses allusions, plus ou moins malveillantes (45 items, 14,20\%) - on trouve ici tout autant cuntstubble ou orifice que our friend with the talking brooch et dickless Tracy ou johndarm. On ne s'étonnera pas de ce que la composition concerne 39 items (12,30\%) ; on sait ce procédé extrêmement productif en anglais, toujours en combinaison avec une matrice sémantique ici (three-bullet Joe, man in blue, bald tire bandit se classent ici, comme yellow leg, cook's own ou beaksman et orderly daughters). La déformation $(10,10 \%, 32$ items) rend compte des divers argots à clefs, en particulier le rhyming slang (grasshopper, lollipop, hot scone, bottle (and stopper), etc.). Enfin, la suffixation, particulièrement productive en anglais, et la troncation représentent respectivement 31 et 30 items $(9,8$ et 9,5\%) (ploddite, flattie, copper - cop, flat, woody, D). Les autres matrices interviennent de façon marginale; on constate sans surprise que les matrices formelles qui permettent le déplacement, c'est-à-dire le changement de catégorie ou la modification d'aspect sont privilégiées.

77 items $(24,4 \%$ du corpus $)$ n'entrent dans aucune matrice formelle: ils se rattachent uniquement à une matrice sémantique, sont seulement remotivés. Des mots comme (raw) lobster (outre la couleur, les pinces jouent un rôle, bien sûr), badge, clown, bacon, finger, beast ou mallet se sont simplement vus affecter un nouveau sens par les argots en raison d'un sème qui, grossi dans l'effet de loupe, a servi de sème de transfert. On constate alors que $86 \%$ des mots du corpus entrent dans une équation sémantique et l'on voit que 191 mots $(60,4 \%)$ montrent une combinaison d'au moins une matrice sémantique avec au moins une matrice formelle. Cette proportion est tout-à-fait 
comparable à ce qui ressort de l'analyse du corpus français du même type que celui examiné ici (61\%), tout comme les autres chiffres. ${ }^{16}$

Si l'on se penche de plus près sur les matrices sémantiques (auxquelles se rattache la catégorie "archétypes» examinée plus haut), l'on constate que la «forme» (équations métonymiques) regroupe 121 items (37,6\% du corpus), en comparaison avec 108 mots $(33,54 \%)$ pour la «fonction » (équations métaphoriques). Du côté des équations métonymiques, comme dans le cas du français, l'aspect physique (largement, l'uniforme et les accessoires du métier, 55 items, 17\%) et les moyens de locomotion (marche et référence à un véhicule, 26 items, 8\%) fournissent de nombreux traits qui sont haussés pour représenter à eux seuls le policier: la couleur de l'uniforme en particulier, avec 21 items (blue-bottle, orange crush, blue meanies, red coat, etc.), des accessoires divers (badge bandit, Brenda Bracelets, button, shades, etc.) ; la marche, du policier qui fait sa ronde (image typique du bobby: flatfoot, plodder, clod hopper, blister foot). L'anglais se distingue du français en attribuant au policier deux traits de caractère qui le désignent métonymiquement: la bêtise (35 items, 10,3\% : defective, Feebie, fly, woodentop, zombie) et la tendance à être corruptible (5 items, 1,5\% : filth, Babylon, goon squad) - en français, si un trait de caractère comme la méchanceté ou la dissimulation peut apparaître, il découle d'une des fonctions du policier.

Ces fonctions se superposent pour les deux langues, mais, si la fonction "protéger » est négligeable des deux côtés $(2,4 \%$ en anglais et $3 \%$ en français) et la fonction "rechercher/arrêter » est très sollicitée (45 items, 13,3\% du corpus anglais ; 14\% en français) avec une domination de la matrice "policier = celui qui attrape " (claw, charperer, Uncle $\mathrm{Nab}(\mathrm{s})$, arm of the law, rosser, etc.), en revanche, la fonction "surveiller/veiller, épier", même importante, est moins productive en anglais qu'en français (55 items, 12,7\% du corpus anglais, 74 (24,1\%) en français. Le sème |œil| (le policier surveille ou épie, et donc le regard, l'œil, est un des attributs de sa fonction) est nettement moins sollicité qu'en français (eye, gazer, peeper) alors qu'il concerne $10 \%$ du corpus français; le sème |odorat| donne lieu à davantage de métaphores en anglais, en particulier autour de pig (oink, lard bacon, bacon, hog, squealers, etc.) ; le sème (délation, traîtrise| (celui qui surveille est un traître potentiel, un mouchard) apparaît moitié moins fréquemment en anglais qu'en français (4,7\% contre 9\%). Cela explique aussi pourquoi la catégorie " archétypes » est si fournie an anglais, où elle représente 19,5\% du corpus $(3,3 \%$ en français). Un tableau de synthèse des matrices sémantiques en anglais et en français figure en annexe (tableau 3).

Tous les items du corpus lexical présenté ici (et il en va de même pour le corpus français parallèle) s'inscrivent dans un nombre fini de matrices formelles et sémantiques, en combinaison dans une majorité des cas; dans presque neuf cas sur dix (huit sur dix en français) une équation métonymique ou métaphorique intervient. La liste de ces équations est établie en isolant le sème mis en jeu (le sème principal dans quelques cas où plusieurs sèmes semblent être sollicités). Ce sème appartient à l'objet considéré, ici le policier ; par un effet de loupe, il est d'emblée privilégié comme s'il était

16 En français: déplacement sémantique seulement: 26,3\% du corpus; mots issus d'équation sémantique : 87,9\% ; déplacement formel uniquement : 12,7\% (plus élevé en français, où les argots à clef et les suffixations argotiques fantaisistes ou non sont plus productifs). Voir Antoine [2011]. 
le seul pertinent, et, dans le cas de la métonymie, cela suffit pour activer et justifier l'équation entre l'objet (policier) et un lexème associé au sème en question. Selon les lieux, les époques, les locuteurs, des sèmes différents, dans une gamme cependant limitée, peuvent être ainsi haussés, et des lexèmes différents peuvent naturellement être activés. Dans le cas de la métaphore, le même mécanisme se produit, mais un double effet de loupe permet le saut métaphorique : le sème privilégié comme seul pertinent de l'objet est aussi repéré de la même façon dans un deuxième objet et le lexème qui désigne le deuxième objet peut alors désigner le premier ${ }^{17}$. Des sèmes différents peuvent donc bien sûr apparaître ici, et la liste en est close tout comme dans le cas de la métonymie. Il est remarquable que, du côté de la métonymie, près de quatre items sur dix $(37,5 \%)$ se rangent par l'effet de loupe sous huit sèmes régulièrement sollicités au cours des siècles; plus de cinq items sur dix (53\%) se rangent par un double effet de loupe, par métaphore, donc, sous huit autres sèmes eux aussi régulièrement sollicités au cours des siècles. L'image mentale globale du policier est stable; seules ses «traductions » varient. On souligne que le français présente à peu près les mêmes caractéristiques $(34,3 \%$ et $42,3 \%$ respectivement), avec un rapport là aussi favorable au double effet de loupe.

L'analyse de ce corpus permet de dire que les désignations à venir du policier s'inscriront dans les mêmes équations, même s'il n'est pas possible de prédire leurs réalisations, bien sûr. L'étude en parallèle des corpus lexicaux des deux langues a des applications évidentes dans le domaine de la lexicographie bilingue (car elle permet d'affiner la représentation dans les dictionnaires des mots considérés et d'apporter des traductions, ou des équivalences, plus pertinentes et diversifiées) et dans le domaine de la traduction (où les choix de traduction sont éclairés par la comparaison des effets de loupe dans les deux langues).

À la lumière des argots, où spontanéité, créativité et immédiateté concourent à l'économie, la métaphore apparaît bien comme un procédé économique autant dans son résultat, qui est condensation, que dans son mécanisme, qui est ce que je définis comme " effet de loupe », forme de condensation là aussi, puisqu'il a pour effet de concentrer, de condenser toute la réalité d'un objet dans un trait, un sème de cet objet traité comme si l'objet n'était que lui. L'argot, un peu comme ailleurs la caricature ou la parodie, se saisit immédiatement d'un sème, vu comme saillant, à qui d'ailleurs il donne toute saillance, qui oblitère les autres sèmes, les rejette en arrière-plan. Cela dit qu'il n'y a pas parcours du sémème puis sélection d'un sème, qu'on l'appelle ainsi ou «feuilletage de l'encyclopédie », pour reprendre la métaphore d'U. Eco. Cela n'est pas dire que les réseaux n'existent pas, que le «feuilletage de sens" (au sens de superposition, cette fois) n'existe pas : l'effet de loupe sur un sème ne signifie pas oubli pur et simple du reste - celui-ci a une « histoire » avec le sème haussé, et les influences sémantiques ou formelles peuvent naturellement persister, renforçant l'effet de loupe ou l'influençant.

\footnotetext{
17 Dans le cas où le sème fait référence à un procès, comme j'ai tenté de le montrer plus haut, si un lexème désignant l'agent existe déjà, le double effet de loupe est simple. Sinon, ce lexème, qui peut être considéré comme en puissance dans le verbe, apparaît par suffixation en -ER (on notera que dans de nombreux dictionnaires unilingues anglais, est souvent enregistrée sous un verbe la forme 'vb-ER', sans définition ou avec la simple définition 'one that vb[es]' (type 'one that snatches').
} 


\section{Annexes}

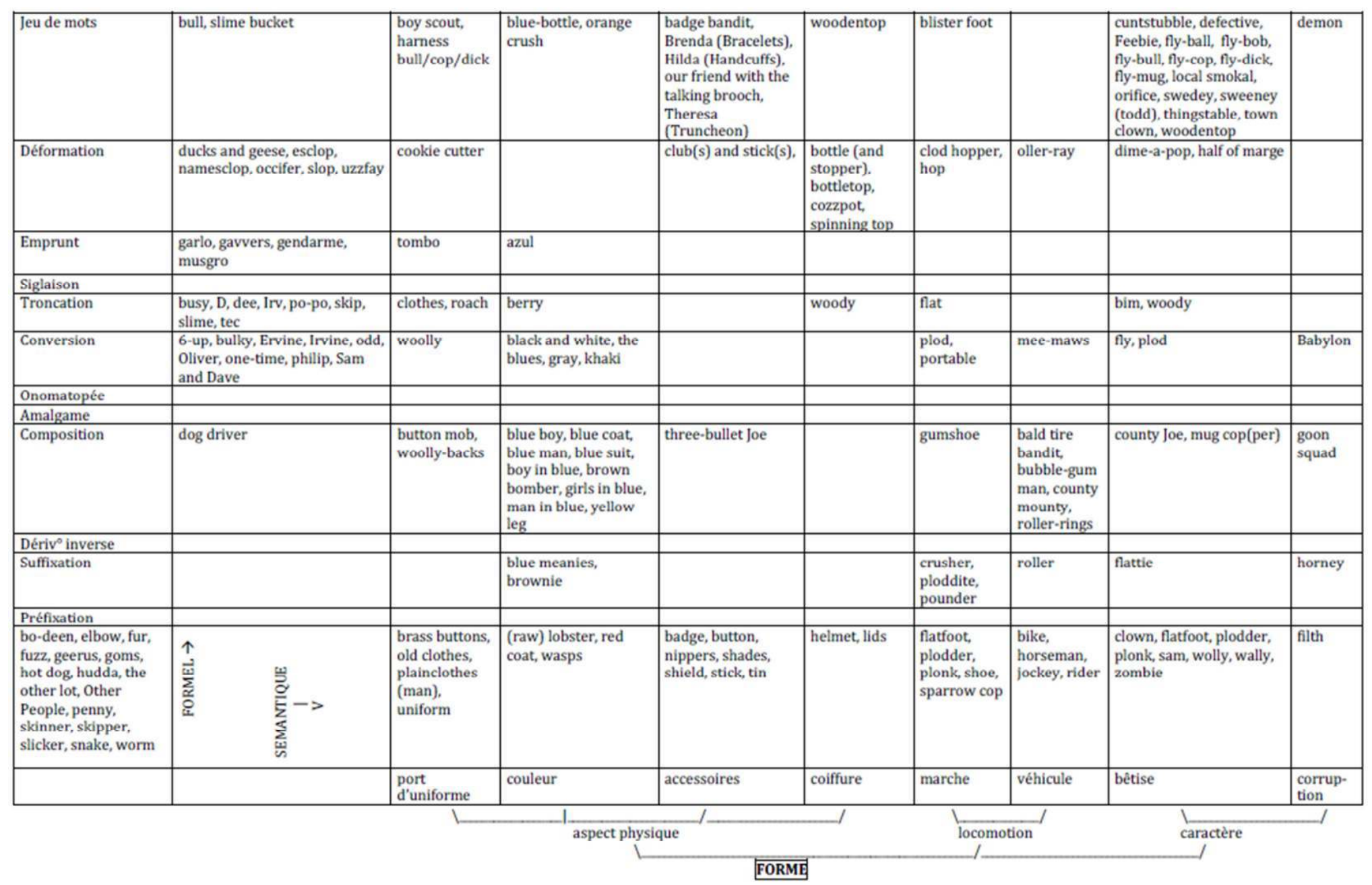

Tableau 1 - Matrices formelles et sémantiques de policeman : équation métonymique, "forme " 


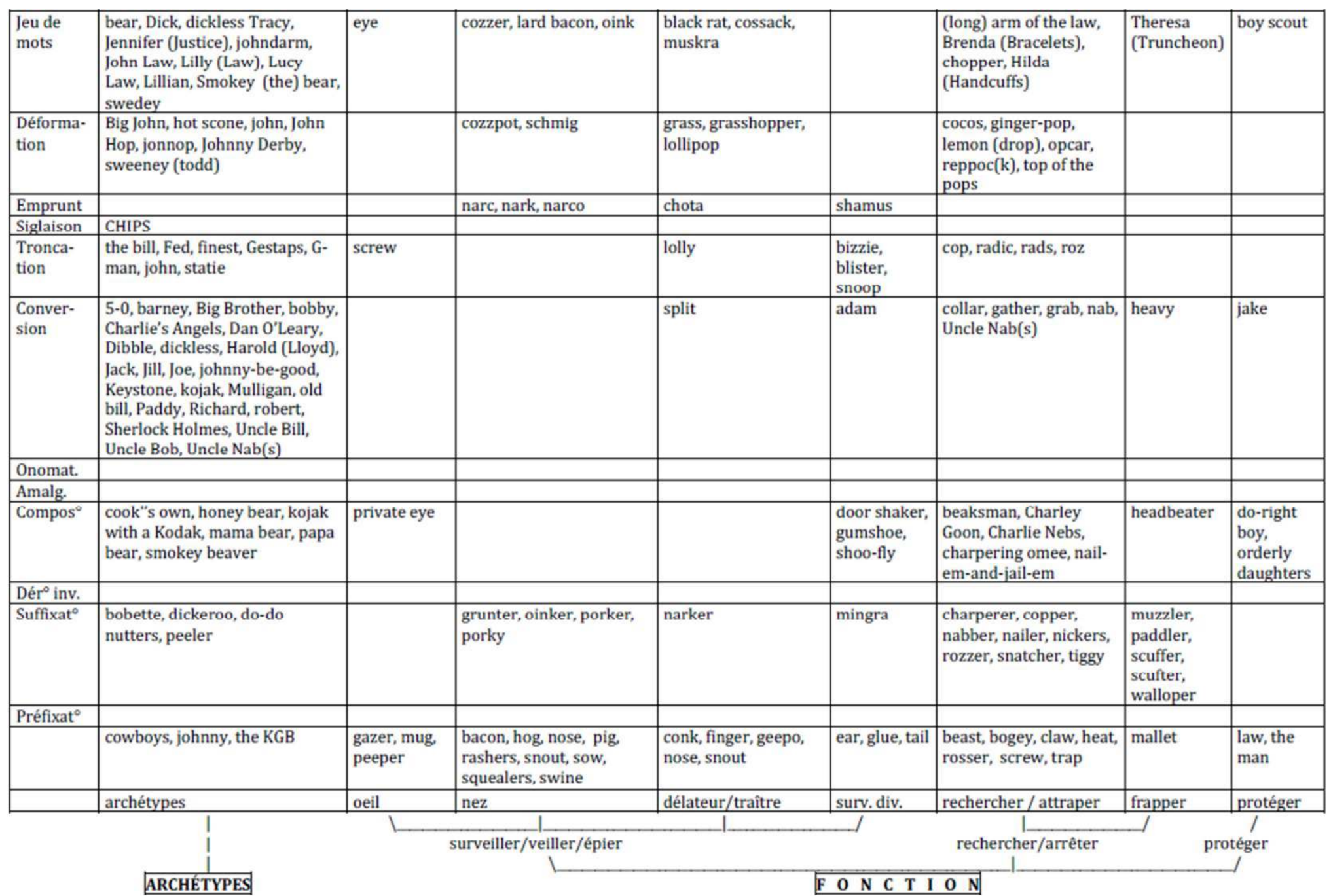

Tableau 2 - Matrices formelles et sémantiques de policeman : équation métaphorique, "fonction » 

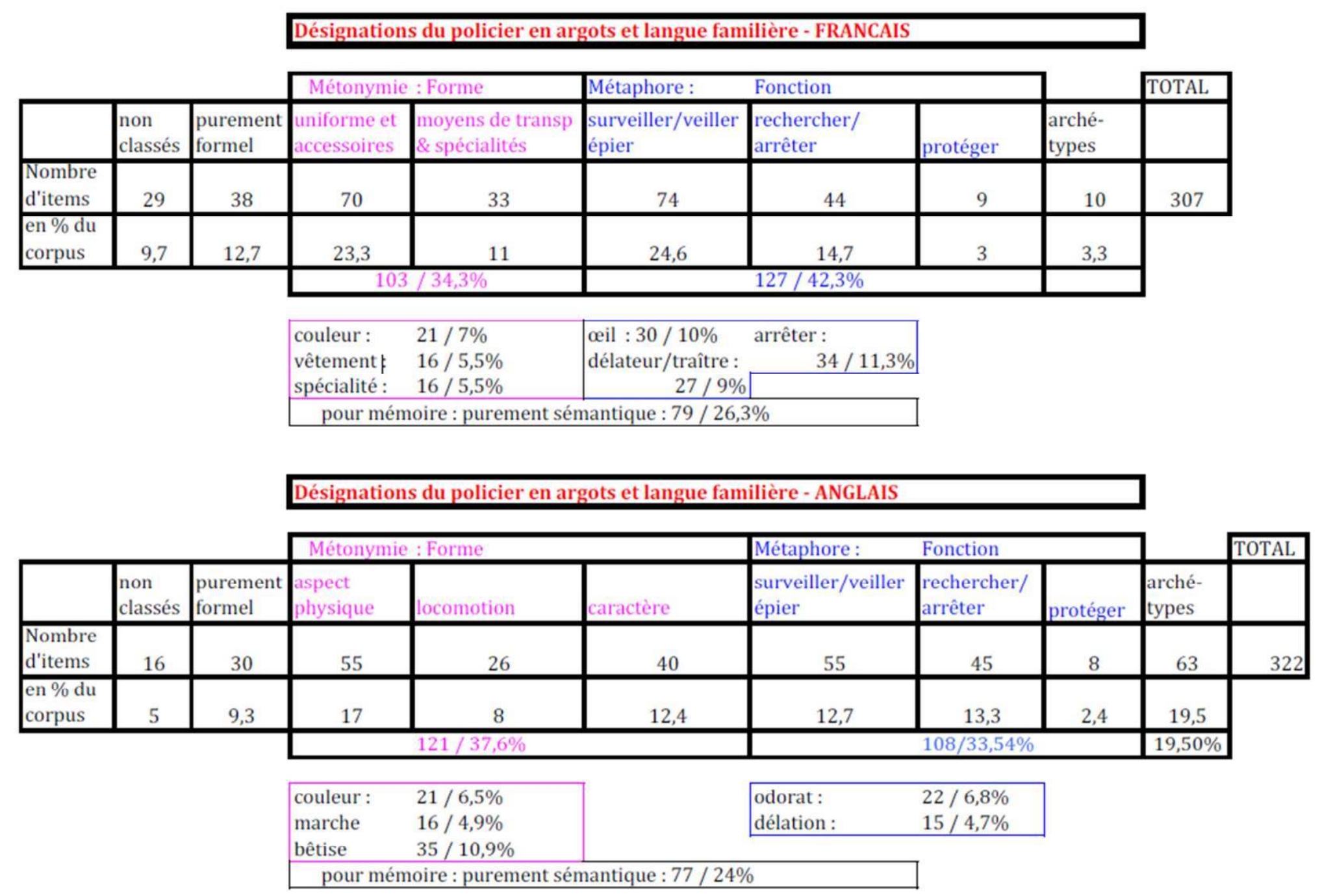

Tableau 3 : Synthèse des matrices sémantiques de policeman et policier 


\section{Bibliographie}

AnToInE Fabrice, « Verlan français, backslang anglais, etc. », Cahiers de lexicologie (CNRS) 74-1, 1999 : 171-183

ANToINE Fabrice, "Argots et langue familière : quelle représentation en lexicographie bilingue?» in ANTOINE Fabrice (éd.), Argots, langue familière et accents en traduction, Lille : Cahiers de la Maison de la Recherche, Ateliers 31, 2004 : 11-23.

ANToINE Fabrice, "De la thune à la caillasse: argent, argots et langue familière » in VatanPour Sina (éd.), L'argent, Villeneuve d'Ascq : Collection UL3, Université Lille 3, $2008: 329-339$.

Antolne Fabrice, «Argots : histoires de métaphores et de métamorphoses. », Les Cahiers du Dictionnaire 3, 2011 : 63-82.

AYTo John, The Oxford Dictionary of Slang, Oxford: OUP, 1998.

BEALE Paul, A Concise Dictionary of Slang and Unconventional English (From A Dictionary of Slang and Unconventional English by Eric Partridge, London: Routledge: 1989.

Botet Serge, Petit traité de la métaphore - Un panorama des théories modernes de la métaphore, Strasbourg: Presses Universitaires de Strasbourg, 2008.

CALvET Louis-Jean, L'argot, Paris : PUF, 1994.

CARADEC François, N'ayons pas peur des mots, Paris : Larousse, 1988.

CARADEc François \& PouY Jean-Bernard, Dictionnaire du français argotique et populaire, Paris : Larousse, 2009.

Colin Jean-Paul \& MEvel Jean-Pierre, Dictionnaire de l'argot, Paris : Larousse, 1990.

Dalzell, Tom \& Victor Terry, The Concise New Partridge Dictionary and Unconventional English, London: Routledge, 2008.

Delvau Alfred, Dictionnaire érotique moderne, Paris : UGE, 1997 [1864].

Dolllon Albert, Dico du sexe, Paris : Fayard, 2002 (repris dans Dictionnaire de l'argot, Paris, Laffont, Collections Bouquins, 2010).

DürRenMatT Jacques, La Métaphore, Paris : Honoré Champion, 2002.

Eco Umberto, "The Scandal of Metaphor: Metaphorology and Semiotics", Poetics Today 4:2, $1983: 217-57$.

ESNAULT Gaston, Métaphores occidentales - Essai sur les valeurs imaginatives concrètes du français parlé en Basse-Bretagne comparé avec les patois, parlers techniques et argots français, Paris : PUF, 1925.

ESNAULT Gaston, Dictionnaire historique des argots français, Paris : Larousse, 1965.

GuIRAuD Pierre, Dictionnaire historique, stylistique, rhétorique, étymologique, de la littérature érotique, Paris : Payot, 1978.

Henry Albert, Métonymie et métaphore, Paris : Klincksieck, 1971.

LAKOFF George \& JoHnson Mark, Les métaphores dans la vie quotidienne (Metaphors We Live by), Paris : Les Éditions de Minuit (1985) 1980.

LE GuERN Michel, Sémantique de la métaphore et de la métonymie, Paris : Larousse, 1973.

LEHMANN, Alise \& BERTHET Françoise, Introduction à la lexicologie - Sémantique et morphologie, Paris : Dunod, 1998.

MARTIN Robert, Pour une logique du sens, Paris : PUF, 1983.

Perret Pierre, Le Nouveau petit Perret illustré par l'exemple, Paris : Jean-Claude Lattès, 1982. 
PotTiER Bernard, "Vers une sémantique moderne», Travaux de linguistique et de littérature II, 1964 : 121-125.

RASTIER François, Sémantique interprétative, Paris : PUF, 1987.

RASTIER François, "Enjeux épistémologiques de la linguistique de corpus », conférence aux deuxièmes Journées de Linguistique de Corpus, Lorient, septembre 2002, http://www.revue-texto.net/Inedits/Rastier/Rastier_Enjeux.html

TourNiER Jean, Introduction descriptive à la lexicogénétique de l'anglais contemporain, Paris-Genève : Champion-Slatkine, 1985.

VIGERIE Patricia, Dictionnaire des gros mots, Lausanne : Favre, 2004.

WENTWORTH Harold \& FLEXnER Stuart B., Dictionary of American Slang, New York: Crowell, 1960. 
(C) Lexis 2014 